\title{
Hysteresis in Asymmetrical GMI Effect in Amorphous Microwires
}

\author{
J. KRAVČÁK*
}

Department of Physics, Faculty of Electrical Engineering and Informatics, Technical University of Košice, Park Komenského 2, 04200 Košice, Slovakia

The double-peak behaviour of giant magneto-impedance (GMI) dependence on the external magnetic field strength $H$ is observed in as-cast glass-covered amorphous thin wire $\mathrm{Co}_{70.5} \mathrm{Fe}_{4.5} \mathrm{Si}_{15} \mathrm{~B}_{10}$ (microwire), as well as in microwire with the removed glass cover. The position of sharp peaks is symmetrical $\left(H= \pm H_{m}\right)$ with respect to zero external magnetic field strength $(H=0)$ and corresponds to the critical field of irreversible magnetization rotation. The maximum GMI ratio $(\Delta Z / Z)_{\max }$ dependence on the amplitude of current $i_{a c}$ at frequency of $1 \mathrm{MHz}$ is analysed by means of the helical anisotropy $\left(0<\alpha<90^{\circ}\right)$, induced during preparation of the microwire and after glass-cover removing. The additional dc bias current $i_{d c} \leq i_{a c}$ is applied in order to achieve asymmetrical GMI effect. Asymmetrical GMI dependences exhibit hysteresis which can be explained by irreversible magnetization rotation at ferromagnetic surface of the microwire.

DOI: $10.12693 /$ APhysPolA.126.132

PACS: $75.30 . \mathrm{Gw}, 75.50 . \mathrm{Kj}, 75.60 . \mathrm{Ej}$

\section{Introduction}

As cast glass-covered ferromagnetic amorphous thin $\mathrm{Co}_{70.5} \mathrm{Fe}_{4.5} \mathrm{Si}_{15} \mathrm{~B}_{10}$ wire (microwire) of a diameter of $17.8 \mu \mathrm{m}$ with small negative magnetostriction has been prepared by Taylor-Ulitovski technique [1]. The relatively small negative magnetostriction results in the creation of a wide, almost circularly magnetized shell domain structure and a narrow axially magnetized core [2]. The preferential orientation of the spontaneous magnetization (magnetic anisotropy) in the microwire is given by magnetostriction and shape anisotropy. Different mechanical properties of the ferromagnetic metallic central part and of the glass cover of the microwire are responsible for deviation of spontaneous magnetization from circumferential (circular) direction in the shell of the microwire (helical magnetic anisotropy) [3]. Additional removing of the glass cover gives the possibility to decrease the helical anisotropy. Considering giant magnetoimpedance (GMI) effect, which is mainly a surface effect, it is very sensitive to the rotation of magnetization in the shell of a microwire [4]. GMI measurements are often used to determine surface magnetic properties of cobalt based microwires. A quasistatic model based on the minimization of the free energy of domain structure was developed [5] to explain the existence of peaks in the GMI dependence in Fig. 2, if only a reversible magnetization rotation is present.

\section{Experimental motivation}

The scanning electron microscopy (SEM) image (Fig. 1a) of the mechanically removed glass cover reveals its weak bonding with the metallic central part of the microwire. Nevertheless the local isolated glass fragments remain strongly fixed to metallic surface (Fig. 1b). It is

*e-mail: jozef.kravcak@tuke.sk possible to deduce a complex inhomogeneous distribution of radial, axial and torsional mechanical stresses in the metallic part of the microwire, induced during its preparation procedure, as well as after glass cover removing. The angle $\alpha$ of deviation of the easy axis of magnetization from the circumferential direction of the microwire (spiral angle, $0<\alpha<90^{\circ}$ ) determines the shape of the hysteresis loop of the microwire during its magnetization along $z$-axis with irreversible magnetization rotation at the critical field [6].
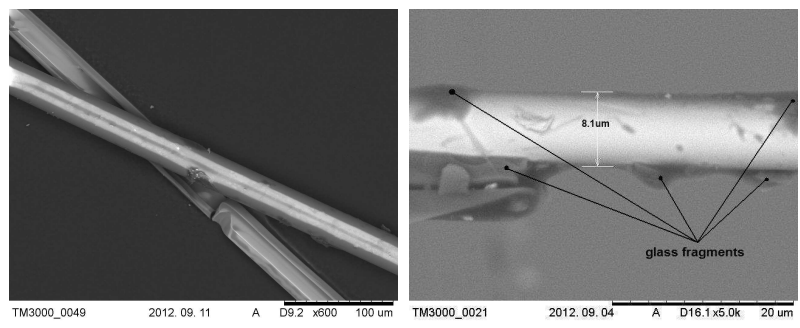

Fig. 1. SEM image of removed glass cover (left) and ferromagnetic amorphous $\mathrm{Co}_{70.5} \mathrm{Fe}_{4.5} \mathrm{Si}_{15} \mathrm{~B}_{10}$ microwire of a diameter $d=8.1 \mu \mathrm{m}$, without glass cover (right).

\section{Experimental results}

The $\operatorname{GMI}(H)$ ratio is usually defined as $\Delta Z / Z=$ $\left(|Z(H)|-\left|Z\left(H_{\max }\right)\right|\right) /\left|Z\left(H_{\max }\right)\right|$, where $|Z|$ is the impedance modulus and $H_{\max }=10 \mathrm{kA} \cdot \mathrm{m}^{-1}$ is the maximum of the applied static axial field $H$, at which the sample is considered to be magnetically saturated along the $z$-axis. In the presented experiments the GMI ratio at the selected frequency of $1 \mathrm{MHz}$ was measured at different amplitudes $i_{a c}$ of the harmonic current flowing through the as-cast $\mathrm{Co}_{70.5} \mathrm{Fe}_{4.5} \mathrm{Si}_{15} \mathrm{~B}_{10}$ microwire. From $\operatorname{GMI}(H)$ ratio dependences, which exhibit a double-peak behaviour (as in Fig. 2), the maximum value of GMI ratio $(\Delta Z / Z)_{\max }$ and peak positions $-H_{m}$ and $+H_{m}$ were plotted as functions of amplitudes $i_{a c}$ in Fig. 3. The 


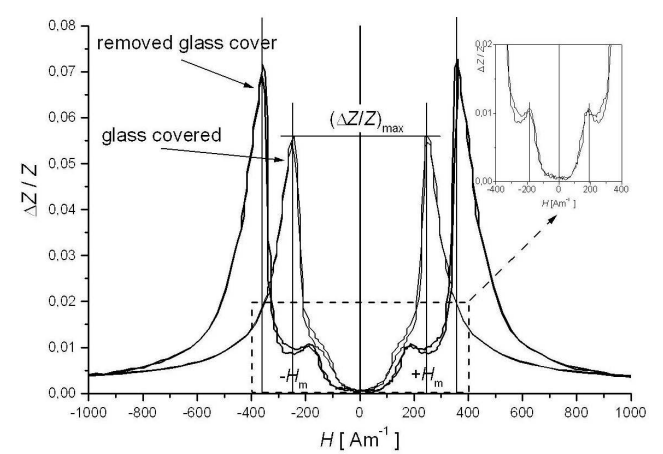

Fig. 2. GMI dependence measured at the frequency of $1 \mathrm{MHz}$ and at the amplitude $i_{a c}=1 \mathrm{~mA}$ in as-cast $\mathrm{Co}_{70.5} \mathrm{Fe}_{4.5} \mathrm{Si}_{15} \mathrm{~B}_{10}$ microwire with glass cover and after glass cover removing.

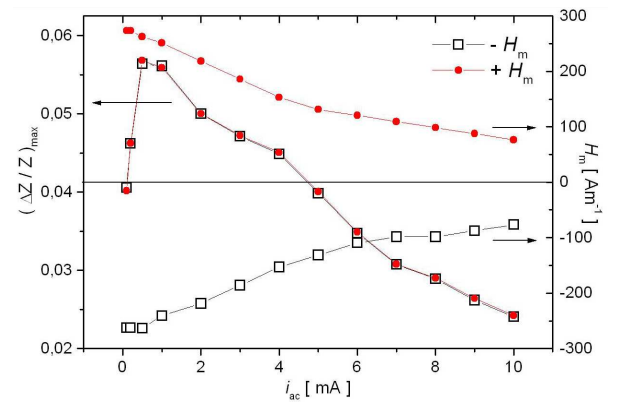

Fig. 3. Maximum value of GMI ratio $(\Delta Z / Z)_{\max }$ (left) and peak positions $-H_{m}$ and $+H_{m}$ (right) as functions of amplitude $i_{a c}$, determined from GMI dependences in $\mathrm{Co}_{70.5} \mathrm{Fe}_{4.5} \mathrm{Si}_{15} \mathrm{~B}_{10}$ microwire, measured at the frequency of $1 \mathrm{MHz}$, as in Fig. 2.

application of an additional dc bias current $i_{d c}$ in the interval $0 \leq i_{d c} \leq i_{a c}$ together with the $i_{a c}=1 \mathrm{~mA}$, revealed an asymmetric character of the $\operatorname{GMI}(H)$ dependences measured in as-cast $\mathrm{Co}_{70.5} \mathrm{Fe}_{4.5} \mathrm{Si}_{15} \mathrm{~B}_{10}$ microwire after glass-cover removing (displayed in Fig. 4).

\section{Discussion}

The measured asymmetrical $\operatorname{GMI}(H)$ dependences of as-cast $\mathrm{Co}_{70.5} \mathrm{Fe}_{4.5} \mathrm{Si}_{15} \mathrm{~B}_{10}$ microwire with glass cover [7] and after glass cover removing (Fig. 4) displays doublepeak behaviour. The theoretical explanation is that for very low amplitudes $i_{a c}$ (or circular field strength $\left.h_{\varphi}=i_{a c} / \pi d\right)$ any reversible domain wall motion at higher frequencies $(\geq 1 \mathrm{MHz})$ is negligible due to strong damping process [8], and magnetization rotation takes place only in the shell of the microwire. The positions of the pair of sharp peaks $\left(H= \pm H_{m}\right)$ are always symmetrical with respect to zero external magnetic fields strength $H=0$ and correspond to the critical field of irreversible magnetization rotation. The dispersion of the critical field together with local variation of the angle $\alpha$ (the easy axis direction) affect the peaks shape.

Removing of the glass cover reduces tensile stresses in the microwire, changes the induced helical anisotropy and the angle $\alpha$. This results in the increase of the critical field and of the maximum value of GMI ratio $(\Delta Z / Z)_{\max }$ in Fig. 2. A residual domain structure formed around local pinning centres of the glass fragments on the microwire surface, manifests itself in formation of a secondary small GMI peaks (inset in Fig. 2).

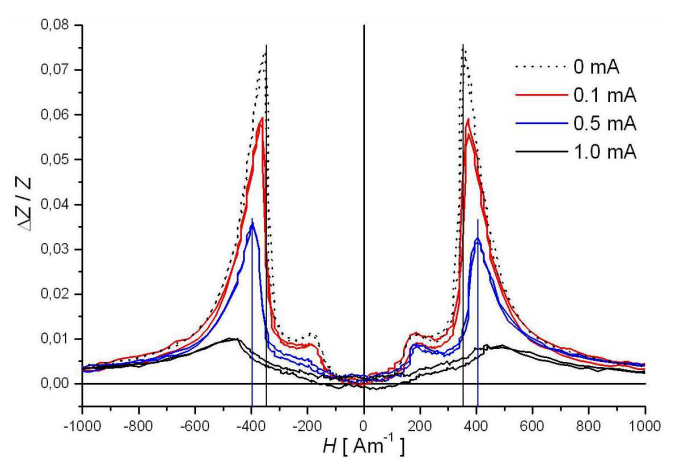

Fig. 4. The asymmetrical GMI effect in $\mathrm{Co}_{70.5} \mathrm{Fe}_{4.5} \mathrm{Si}_{15} \mathrm{~B}_{10}$ microwire after glass cover removing, measured at frequency of $1 \mathrm{MHz}$ with amplitude $i_{a c}$ of $1 \mathrm{~mA}$ and applied dc bias current $i_{d c}$ was varied from 0 to $1 \mathrm{~mA}$.

\section{Conclusions}

Generally the application of bias $i_{d c}$ (or static circular field of strength $\left.H_{\varphi}=i_{d c} / \pi d\right)$, significantly decreases $(\Delta Z / Z)_{\max }$, and brings asymmetry in $\operatorname{GMI}(H)$ ratio, when the static axial field $H$ has parallel or antiparallel orientation with respect to direction of bias $i_{d c}$.

Despite the fact that occurrence of the hysteresis in $\operatorname{GMI}(H)$ dependences is a disadvantage from the application point of view, it introduces a valuable information about the magnetization dynamics and the magnetic behaviour of surface domain structure.

\section{Acknowledgments}

This work was supported by Slovak Research and Development Agency under the contract No. APVV-002711. This paper was developed within the project "Centre of Excellence for Integrated Research \& Exploitation of Advanced Materials and Technologies in Automotive Electronics" ITMS 26220120055.

\section{References}

[1] M. Vázquez, Physica B 299, 302 (2001).

[2] L.V. Panina, K. Mohri, T. Uchiyama, M. Noda, IEEE Trans. Magn. 31, 1249 (1995).

[3] A. Chizhik, J.M. Blanco, A. Zhukov, J. Gonzalez, G. Garcia, P. Gawronski, K. Kulakowski, IEEE Trans. Magn. 42, 3889 (2006).

[4] M. Knobel, M. Vázquez, L. Kraus, in:Handbook of Magnetic Materials, 15, Ed. by K. H. J. Buschow, Elsevier Science B.V., 2003, p. 497.

[5] F.L.A. Machado, S.M. Rezende, J. Appl. Phys. 79, 6558 (1996).

[6] S. Chikazumi, Physics of Magnetism John Wiley \& Sons, New York 1964, p. 281.

[7] J. Kravčák, R.Varga, Acta Phys. Pol. A 118, 762 (2010).

[8] N.A. Usov, A.S. Antonov, A.N. Lagarkov, J. Magn. Magn. Mater. 185, 159 (1998). 